\title{
A combination of MSH2 DNA mismatch repair deficiency and expression of the SV40 large $T$ antigen results in cisplatin resistance of mouse embryonic fibroblasts
}

\author{
SHARI-LYNNE YASIN and ANDREW J. RAINBOW \\ Department of Biology, McMaster University, Hamilton, Ontario L8S 4K1, Canada \\ Received February 28, 2011; Accepted April 8, 2011
}

DOI: $10.3892 /$ ijo.2011.1065

\begin{abstract}
Mutations in the human mismatch repair (MMR) genes are associated with hereditary non-polyposis colorectal cancer as well as other sporadic cancers. MMR gene mutations have been implicated in the resistance of human tumours to cisplatin and several tumour-derived MMR-deficient cells show cisplatin resistance in vitro. In addition, hypoxia, a common feature of the tumour microenvironment, has been shown to influence tumour responses to conventional cancer treatments. We have examined the role of the $\mathrm{mMSH} 2 \mathrm{MMR}$ protein on repair of cisplatin-damaged DNA and cisplatin sensitivity in mMSH2-deficient murine fibroblasts and mMSH2-proficient controls under conditions of normoxia and hypoxia. Sensitivity to cisplatin was measured using the MTT assay and clonogenic survival. Repair of cisplatin-damaged DNA was measured using a host cell reactivation (HCR) assay employing a non-replicating recombinant virus expressing the $\beta$-galactosidase reporter gene. Sensitivity to cisplatin was significantly less and HCR of the cisplatin-damaged reporter gene was significantly greater in SV40-transformed mMSH2-deficient cells (MS5-7) compared to mMSH2-proficient controls (BC1-6) under both normoxic and hypoxic conditions. In contrast, sensitivity to cisplatin was significantly greater and HCR was similar in primary mMSH2-deficient compared to mMSH2-proficient murine fibroblasts under both normoxic and hypoxic conditions. Sensitivity to cisplatin was also significantly greater and HCR was similar in primary mMSH2-deficient compared to mMSH2proficient murine fibroblasts transfected with a control plasmid under both normoxic and hypoxic conditions. In contrast, sensitivity to cisplatin was less and HCR was similar in primary mMSH2-deficient compared to mMSH2-proficient murine fibroblasts transfected with a plasmid expressing SV40 large $\mathrm{T}$ antigen under both normoxic and hypoxic conditions. These
\end{abstract}

Correspondence to: Dr Andrew J. Rainbow, Department of Biology, McMaster University, 1280 Main Street West Hamilton, Ontario L8S 4K1, Canada

E-mail: rainbow@mcmaster.ca

Key words: mismatch repair, nucleotide excision repair, host cell reactivation, recombinant adenovirus, cisplatin, hypoxia, SV40 large $\mathrm{T}$ antigen results suggest that loss of MMR alone does not result in increased resistance to cisplatin in murine fibroblasts and that additional concomitant alterations in cells expressing the SV40 large $\mathrm{T}$ antigen are responsible for cisplatin resistance through a modulation of DNA repair capacity and/or apoptosis.

\section{Introduction}

Cisplatin is one of the most effective and commonly used drugs for cancer chemotherapy. It has demonstrated anticancer effects through the formation of bulky DNA crosslinks for a broad spectrum of solid tumours. However, intrinsic and acquired resistance to cisplatin has been observed in a number of cancers posing a major challenge to clinical treatment. The underlying mechanisms of cisplatin resistance are multifactorial. There is evidence that the p53 tumour suppressor and the mismatch repair genes both play an important role with regard to cellular responses to cisplatin. The development of cisplatin resistance is generally associated with the loss of p53 function (1-4), although loss of p53 function has also been reported to result in the sensitization of some cell lines to cisplatin $(5,6)$. Mutations in the human MMR genes $h M L H 1, h M S H 2$ and to a lesser extent $h P M S 2$, account for $90 \%$ of hereditary non-polyposis colorectal cancer and hMSH6 mutations are associated with other sporadic cancers (reviewed in ref. 7). It has been suggested that although the MMR complex cannot repair cisplatin adducts, attempts to repair these adducts by MMR may be made followed by inevitable failure and subsequent apoptosis induction $(1,8)$. Therefore, loss of MMR ability may enable cells to survive DNA damage induced by cisplatin. Indeed, defects in MMR genes have been implicated in the resistance of human tumour cells to cisplatin in vitro (8-12). However, reports of cisplatin resistance in MMR-deficient murine cells in vitro are conflicting and suggest the joint requirement of MMR deficiency and additional gene alterations for cisplatin resistance. For example, Claij and Riele found that the response of $\mathrm{mMSH} 2$-deficient and $\mathrm{mMSH} 2$-proficient mouse embryonic stem cells to cisplatin were similar and restoration of $M s h 2$ activity in deficient cells with cDNA did not increase their sensitivity (13). In addition, Sansom and Clarke have reported only a slightly reduced apoptotic response in MSH2-deficient murine intestinal enterocytes which had no overall effect on survival following cisplatin treatment (14). 
The tumour microenvironment represents another important influence over cellular responses to DNA damaging agents. This microenvironment results from an inadequate blood and oxygen supply and is characterized by regions of fluctuating or chronic hypoxia, low $\mathrm{pH}$ and nutrient deprivation. Studies have shown that cells under hypoxic stress exhibit decreased DNA repair, elevated mutagenesis, downregulation of key repair proteins and abnormal cell physiology $(15,16)$.

In the current work we used the MTT assay and HCR of a cisplatin-damaged reporter gene to examine the effects of MMR loss and hypoxia on cisplatin sensitivity and repair of cisplatin-damaged DNA in MMR-proficient and MMR-deficient isogenic murine embryonic fibroblasts (MEFs). The use of both primary and transformed isogenic murine fibroblasts allowed for a precise determination of the role of MMR in resistance to cisplatin and circumvented some of the difficulties associated with human tumour cells such as additional acquired mutations and chromosome transfer to restore wild-type MMR activity. Additionally, few studies have compared the repair of cisplatin damage in primary versus transformed fibroblasts. Changes induced by transformation with simian virus 40 (SV40) have been reported to have important effects on both activation of the DNA damage response and DNA repair itself (17-20).

We sought to determine whether increased repair of cisplatindamaged DNA in these MMR-deficient cells was concomitant with increased cell survival as several studies have implicated DNA repair as a key mechanism of cisplatin resistance (reviewed in ref. 21). We also sought to determine whether differential effects of hypoxia on repair of cisplatin-damaged DNA in mMSH2-deficient and mMSH2-proficient MEFs could account for increased mutability, since increased mutagenesis has been reported for some cell types under conditions of hypoxia (16).

\section{Materials and methods}

Cells. Primary MEFs expressing wild-type mMSH2 (M2WT) and primary mMSH2 knockout cells (M2KO), as well as SV40transformed MEFs expressing wild-type mMSH2 (BC1-6) and SV40-transformed mMSH2 knockout cells (MS5-7) were obtained from Dr S.E. Andrew at the University of Alberta, Edmonton, AB and have been described previously (22). Cell cultures were grown in a humidified incubator at $37^{\circ} \mathrm{C}$ in $5 \%$ $\mathrm{CO}_{2}$. Cells were maintained in alpha-minimum essential media ( $\alpha$-MEM) supplemented with 20\% (M2WT and M2KO) or $10 \%$ (BC1-6 and MS5-7) fetal bovine serum (Hyclone, USA) and $1 \%$ antibiotic-antimycotic (Gibco, USA).

Four additional cell lines were established from primary M2WT and M2KO cells through transfection with either a control plasmid (pBabe-neo) or a plasmid with a $2.2 \mathrm{~kb}$ SV40 large $\mathrm{T}$ antigen gene insert (pBabe-neo LTag). These cell lines, M2KO pBabe-neo (M2KO Control), M2WT pBabe-neo (M2WT Control), M2KO pBabe-neo LTag (M2KO LTag) and M2WT pBabe-neo LTag (M2WT LTag) were maintained in $\alpha$-MEM supplemented with $15 \%$ fetal bovine serum, $1 \%$ antibioticantimycotic and $200 \mu \mathrm{g} / \mathrm{ml} \mathrm{G} 418$ sulfate (Gibco, NY).

Induction of cisplatin damage to viral DNA. The non-replicating recombinant adenovirus AdCA35 (AdMCMVlacZ) used in these experiments was obtained from Dr F.L. Graham at McMaster University, Hamilton, ON. The viral construct consists of the $E$. coli lacZ reporter gene encoding $\beta$-galactosidase $(\beta$-gal), a murine cytomegalovirus early promoter (MCMV-IE) and a SV40 polyadenylation signal (23).

Cisplatin was purchased as a $3333 \mu \mathrm{M}$ stock solution at $1 \mathrm{mg} / \mathrm{ml}$ (Faulding, QC). Drug concentrations were prepared in a solution of $\alpha$-MEM and low chloride $(50 \mathrm{mM})$ phosphate buffered saline (PBS) for a final chloride concentration of $6.7 \mathrm{mM}$. A viral suspension was also prepared in this low chloride solution and added to each drug dilution for final cisplatin concentrations of $1-8 \mu \mathrm{M}$. The virus preparation was incubated for $12 \mathrm{~h}$ at $37^{\circ} \mathrm{C}$ and DNA damage to the virus was subsequently stopped with the addition of unsupplemented $\alpha$-MEM to each concentration tube.

Host cell reactivation assay. Cells were seeded in 96-well tissue culture plates at densities of $3.0 \times 10^{4}$ cells per well for the primary MEFs and $4.0 \times 10^{4}$ cells per well for the SV40transformed and transfected MEFs. Cells were then incubated for $24 \mathrm{~h}$ at $37^{\circ} \mathrm{C}$ under normoxic conditions $\left(21 \% \mathrm{O}_{2}\right)$. Following incubation, cells were infected with $40 \mu \mathrm{l}$ of cisplatin-treated or non-treated virus at a multiplicity of infection of 40 plaque forming units per cell. After 90 min of viral adsorption, fresh supplemented growth media was added to the wells followed by incubation at $37^{\circ} \mathrm{C}$ under normoxic or hypoxic conditions for $40 \mathrm{~h}$. Hypoxic incubation conditions were established with a continuous flow mixture of $94 \% \mathrm{~N}_{2}, 5 \% \mathrm{CO}_{2}$ and $1 \% \mathrm{O}_{2}$ gas. Cells were harvested and scored for $\beta$-gal activity by the addition of $1 \mathrm{mM}$ CPRG (Roche, IN) in a solution of $0.01 \%$ Triton $\mathrm{X}-100,1 \mathrm{mM} \mathrm{MgCl}$ and $100 \mathrm{mM} \mathrm{Na}_{2} \mathrm{HPO}_{4}+\mathrm{NaH}_{2} \mathrm{PO}_{4}$ at $\mathrm{pH}$ 8.3. $\beta$-gal activity was determined with absorbance measurements at $570 \mathrm{~nm}$ using a 96-well spectrophotometer (EL340 Bio Kinetics Reader, Bio-Tek Instruments). $\beta$-gal saturation curves were plotted and only readings on the linear part of the curves were used for analyses. $\beta$-gal activity was expressed relative to untreated controls $( \pm S E)$ with background levels of $\beta$-gal expression subtracted for each determinant. A minimum of three experiments, each with triplicate determinants, were performed for all cell lines.

Plasmid preparation and transfection of primary murine embryonic fibroblasts. Competent E. coli Top 10 cells, Phoenix amphotrophic retroviral packaging cells, the pBabe-neo control plasmid and the pBabe-neo LTag plasmid expressing SV40 large T antigen were kindly provided by Dr X.-D. Zhu at McMaster University, Hamilton, ON. E. coli cells were transformed with the control plasmid or the plasmid expressing SV40 large T antigen. Both plasmids were isolated from bacterial cultures and purified using a Plasmid Maxi Kit (Qiagen Sciences, VA). Plasmid DNA was then ethanol precipitated and packaged in Phoenix cells through calcium phosphate transfection (reviewed in ref. 24).

Primary M2WT cells and primary M2KO cells were seeded in $10 \mathrm{~cm}$ culture dishes at a density of $1 \times 10^{6}$ cells/dish and incubated at $37^{\circ} \mathrm{C}$ overnight. Over 3 days, cells were repeatedly infected with virus-containing media from Phoenix cell cultures in the presence of $4 \mu \mathrm{g} / \mathrm{ml}$ polybrene (Sigma, MO). Cell lines were then selected for 8 days with supplemented media containing $600 \mu \mathrm{g} / \mathrm{ml} \mathrm{G} 418$ sulfate. Cells were subsequently maintained in supplemented media containing $200 \mu \mathrm{g} / \mathrm{ml}$ G418 sulfate. 

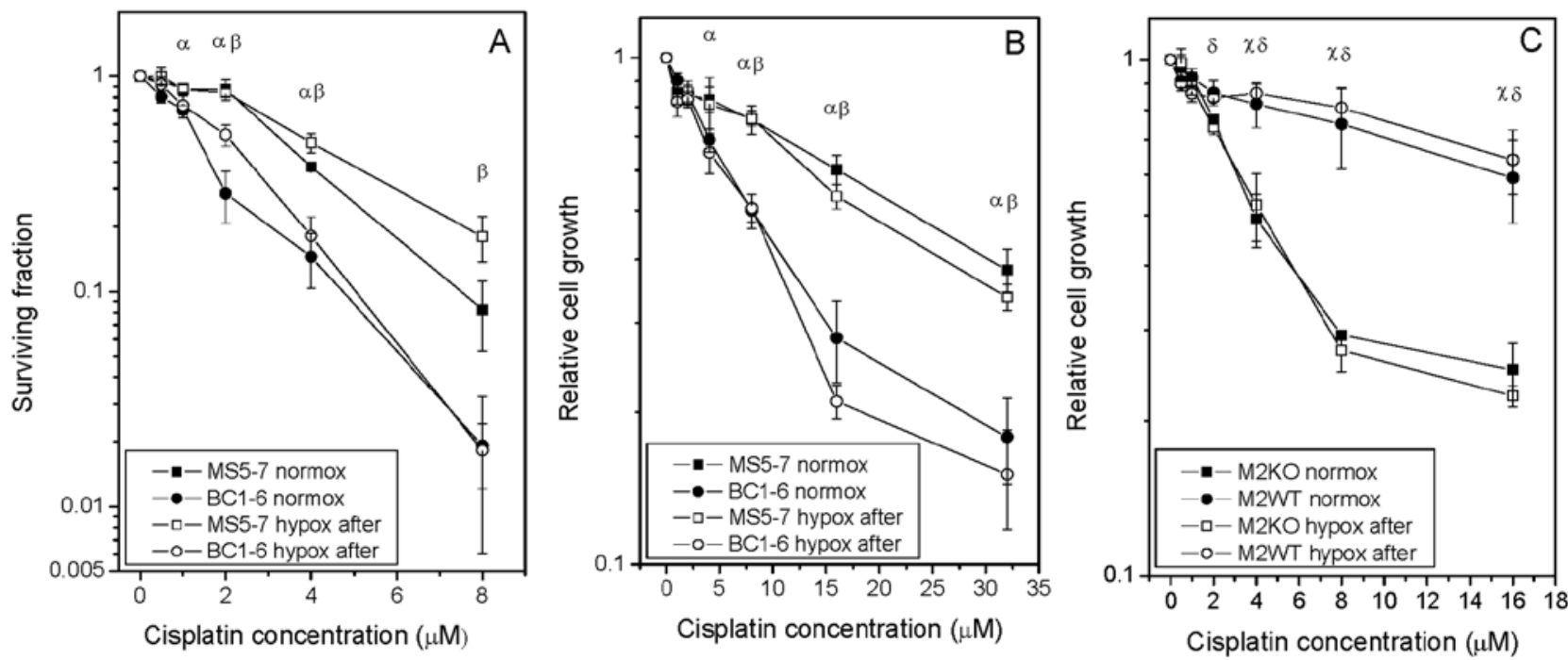

Figure 1. Cisplatin sensitivity of MSH2-deficient and MSH2-proficient murine fibroblasts under normoxic (closed symbols) or hypoxic (open symbols) incubation conditions. (A) Clonogenic survival of cisplatin-treated SV40-transformed MSH2-deficient MS5-7 cells and MSH2-proficient BC1-6 cells. (B) Relative cell growth as determined by the MTT assay for cisplatin-treated SV40-transformed MSH2-deficient MS5-7 cells and MSH2-proficient BC1-6 cells. (C) Relative cell growth as determined by the MTT assay for cisplatin-treated primary MSH2-deficient M2KO and MSH2-proficient M2WT murine fibroblasts. Each point represents the average \pm SE of 3 independent experiments each performed in triplicate. ${ }^{~} \mathrm{MS} 5-7>\mathrm{BC} 1-6$ (normoxia); significant by two-sample t-test, $\mathrm{P}<0.05$. ${ }^{\beta}$ MS5-7 > BC1-6 (hypoxia); significant by two-sample t-test, $\mathrm{P}<0.05 .{ }^{\mathrm{x}} \mathrm{M} 2 \mathrm{KO}<\mathrm{M} 2 \mathrm{WT}$ (normoxia); significant by two-sample t-test, $\mathrm{P}<0.05 .{ }^{\circ} \mathrm{M} 2 \mathrm{KO}<\mathrm{M} 2 \mathrm{WT}$ (hypoxia); significant by two-sample t-test, $\mathrm{P}<0.05$.

MTT reduction assay for cell viability. Cells were seeded in 96-well plates at densities of $2.5 \times 10^{3}$ cells/well (M2WT, M2KO, M2WT Control, M2KO Control, M2WT LTag and M2KO LTag), $1.9 \times 10^{3}$ cells/well (MS5-7) and $1.25 \times 10^{3}$ cells/well (BC1-6). Cells were treated $7 \mathrm{~h}$ later with cisplatin concentrations of 0.5-32 $\mu \mathrm{M}$ in supplemented media or with supplemented media alone for $1 \mathrm{~h}$. Fresh supplemented media was then added to the wells followed by either normoxic incbuation alone or $40 \mathrm{~h}$ hypoxic incubation $\left(1 \% \mathrm{O}_{2}\right)$ and subsequent normoxic incubation. Primary M2KO and M2WT cells were scored for viability 5 days post-treatment and pBabe-neo transfected M2KO Control and M2WT Control cells were scored 4 days post-treatment. In contrast, all cell lines expressing large $\mathrm{T}$ antigen (BC1-6, MS5-7, M2KO LTag and M2WT LTag) were scored 3 days post-treatment given their faster rate of proliferation relative to primary and transfected control cells. MTT [3-(4,5-dimethylthiazol-2-yl)-2,5-diphenyltetrazolium bromide] (Sigma), was prepared at $5 \mathrm{mg} / \mathrm{ml}$ and added to cells followed by incubation at $37^{\circ} \mathrm{C}$ for $4 \mathrm{~h}$. Dimethyl sulphoxide (DMSO), was used to solubilize the formazan product and absorbances were determined at $570 \mathrm{~nm}$ with a 96-well spectrophotometer. Reduction of MTT to formazan depends on active mitochondrial reductase enzymes and therefore, is used to infer cell viability and growth. Cell growth was expressed relative to untreated controls $( \pm \mathrm{SE})$. A minimum of three experiments, each with triplicate determinants, were performed for all cell lines.

Clonogenic survival assay. MS5-7 and BC1-6 cells were seeded at a density of 500 cells/well in 6-well plates and treated $6 \mathrm{~h}$ later with cisplatin concentrations of $0.5-8 \mu \mathrm{M}$ in supplemented media or with supplemented media alone for $1 \mathrm{~h}$. Cells were rinsed in PBS and fresh supplemented media was added to the wells followed by either normoxic incbuation alone or $40 \mathrm{~h}$ hypoxic incubation $\left(1 \% \mathrm{O}_{2}\right)$ and subsequent normoxic incubation. At 6 days post-treatment cells were stained with $0.5 \%$ crystal violet (Sigma) in $70 \%$ ethanol and $10 \%$ methanol. Colonies with $>32$ cells were scored and cell survival was expressed relative to untreated controls $( \pm \mathrm{SE})$. A minimum of three experiments, each with triplicate determinants, were performed for all cell lines.

\section{Results}

Survival of cisplatin-treated MSH2-deficient and MSH2proficient MEFs. Pooled results for the cisplatin sensitivity of mMSH2-deficient and mMSH2-proficient MEFs under normoxic and hypoxic conditions are shown in Fig. 1. Sensitivity to cisplatin was significantly less in SV40-transformed mMSH2deficient cells (MS5-7) compared to mMSH2-proficient controls (BC1-6) under both normoxic and hypoxic conditions as measured by either the clonogenic assay (Fig. 1A) or the MTT assay (Fig. 1B). In contrast, sensitivity to cisplatin was significantly greater in primary mMSH2-deficient compared to mMSH2-proficient murine fibroblasts under both normoxic and hypoxic conditions as measured by the MTT assay (Fig. 1C).

Host cell reactivation in MSH2-deficient and MSH2-proficient $M E F s$. Pooled results for the relative $\beta$-gal activity of the cisplatin-damaged reporter gene at $40 \mathrm{~h}$ after infection with AdCA35 for mMSH2-deficient and mMSH2-proficient MEFs are shown in Fig. 2. Under normoxic conditions the SV40-transformed mMSH2-deficient MS5-7 cells showed a significantly enhanced HCR relative to the $\mathrm{mMSH} 2$-proficient BC1-6 cells (Fig. 2A). In contrast, no significant difference in repair was observed between primary mMSH2-deficient M2KO cells and mMSH2-proficient M2WT cells at $40 \mathrm{~h}$ after infection under conditions of normoxia or hypoxia (Fig. 2B). 

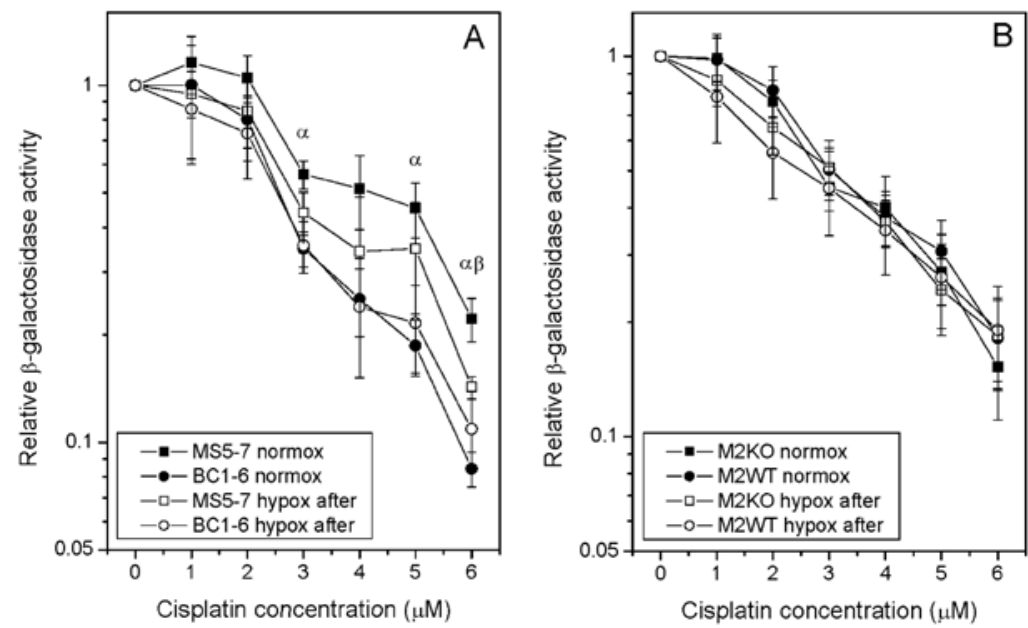

Figure 2. Host cell reactivation of $\beta$-galactosidase activity for the cisplatin-damaged AdCA35lacZ virus in MSH2-deficient and MSH2-proficient murine fibroblasts under normoxic (closed symbols) or hypoxic (open symbols) incubation conditions. (A) SV40-transformed MSH2-deficient MS5-7 cells and MSH2-proficient BC1-6 cells. (B) Primary MSH2-deficient M2KO and MSH2-proficient M2WT murine fibroblasts. Each point represents the average \pm SE of at least 5 independent experiments each performed in triplicate. ${ }^{\alpha} \mathrm{MS} 5-7>\mathrm{BC} 1-6$ (normoxia); significant by two-sample t-test, $\mathrm{P}<0.05$. ${ }^{\beta}$ hypoxia $<$ normoxia (MS5-7); significant by two-sample t-test, $\mathrm{P}<0.05$.
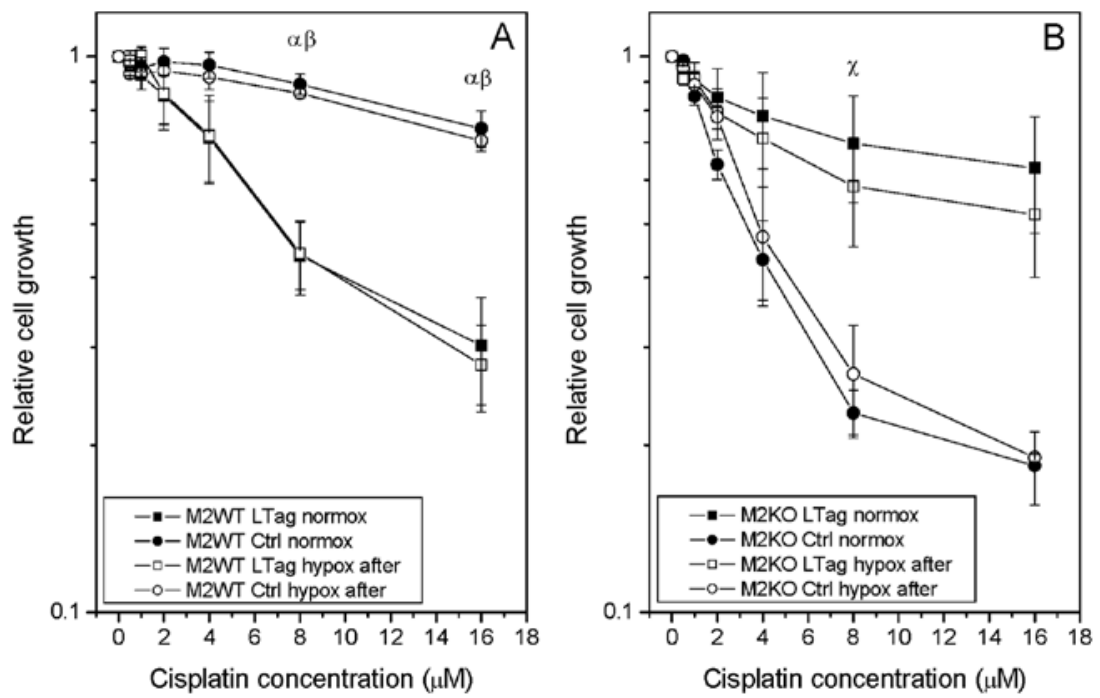

Figure 3. Effects of LTag expression on cisplatin sensitivity of MSH2-proficient M2WT and MSH2-deficient M2KO primary murine fibroblasts under normoxic (closed symbols) or hypoxic (open symbols) incubation conditions. (A) Relative cell growth as determined by the MTT assay for cisplatin-treated MSH2proficient M2WT pBabe-neo LTag cells and M2WT pBabe-neo control cells. (B) Relative cell growth as determined by the MTT assay for cisplatin-treated MSH2-deficient M2KO pBabe-neo LTag cells and M2KO pBabe-neo control cells. Each point represents the average \pm SE of at least 3 independent experiments each performed in triplicate. ${ }^{a} \mathrm{M} 2 \mathrm{WT}$ LTag $<$ M2WT Ctrl (normoxia); significant by two-sample t-test, $\mathrm{P}<0.05 .{ }^{\beta} \mathrm{M} 2 \mathrm{WT}$ LTag $<$ M2WT Ctrl (hypoxia); significant by two-sample t-test, $\mathrm{P}<0.05$. ${ }^{\mathrm{M}} \mathrm{M} 2 \mathrm{KO} \mathrm{LTag}>\mathrm{M} 2 \mathrm{KO} \mathrm{Ctrl}$ (normoxia); significant by two-sample t-test, $\mathrm{P}<0.05$.

Since it was possible that primary M2KO cells had an enhanced rate of repair for cisplatin-damaged DNA compared to M2WT cells that was not detectable when $\beta$-gal activity was scored at $40 \mathrm{~h}$ after infection, we also measured the relative $\beta$-gal activity for the cisplatin-damaged reporter at earlier time points of 12 and $24 \mathrm{~h}$ following infection of the primary $\mathrm{M} 2 \mathrm{KO}$ and M2WT cells. Scoring for $\beta$-gal at 12 and $24 \mathrm{~h}$ after infection did not reveal any significant differences in repair rate between primary M2KO and M2WT cells (data not shown).

Effects of SV4O large T antigen expression on cisplatin sensitivity of MSH2-deficient and MSH2-proficient MEFs. We also examined cisplatin sensitivity by the MTT assay in primary $\mathrm{mMSH} 2$-deficient and $\mathrm{mMSH} 2$-proficient murine fibroblasts that had been transfected with either a control plasmid or a plasmid expressing the SV40 large T antigen. Expression of SV40 large T antigen increased the cisplatin sensitivity in primary mMSH2-proficient murine fibroblasts (Fig. 3A) but decreased the cisplatin sensitivity in primary mMSH2-deficient murine fibroblasts (Fig. 3B). Consistent with the results for non-transfected primary murine fibroblasts (Fig. 1C), the sensitivity to cisplatin was significantly greater in primary mMSH2-deficient compared to mMSH2-proficient murine fibroblasts transfected with the control plasmid under both normoxic and hypoxic conditions (Fig. 4A). In contrast, cisplatin sensitivity was reduced in primary mMSH2-deficient 

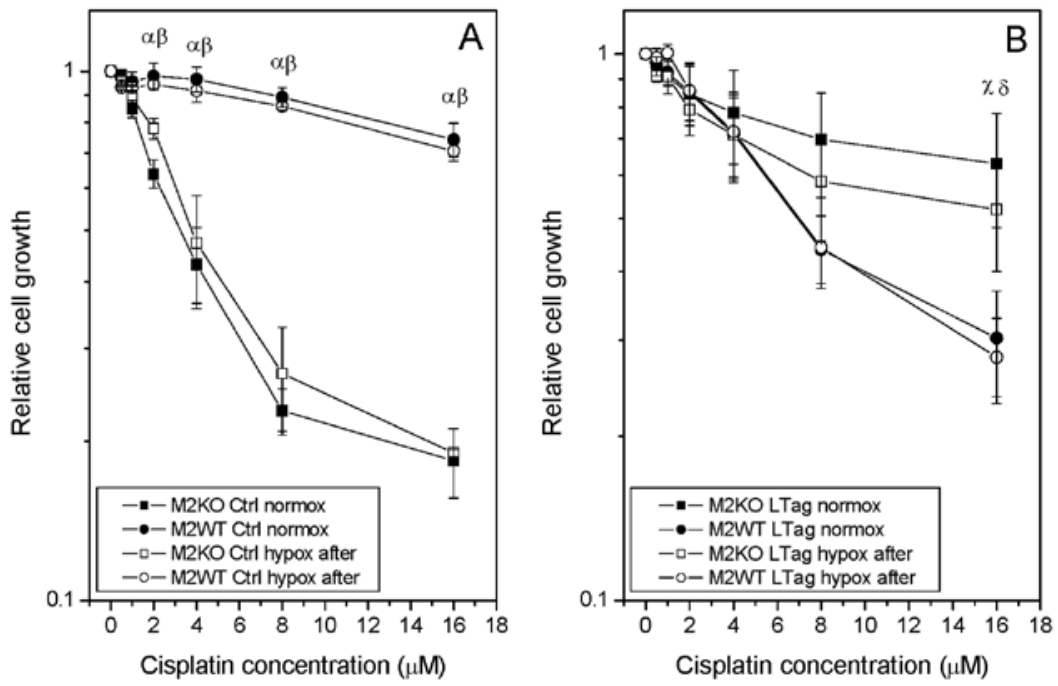

Figure 4. LTag expression alters the relative cisplatin sensitivity of MSH2-proficient M2WT and MSH2-deficient M2KO primary murine fibroblasts under normoxic (closed symbols) or hypoxic (open symbols) incubation conditions. (A) Relative cell growth as determined by the MTT assay of cisplatin-treated MSH2-deficient M2KO pBabe-neo control cells and MSH2-proficient M2WT pBabe-neo control cells. (B) Relative cell growth as determined by the MTT assay of cisplatin-treated MSH2-deficient M2KO pBabe-neo Large Tag cells and MSH2-proficient M2WT pBabe-neo Large Tag cells. Each point represents the average \pm SE of at least 3 independent experiments each performed in triplicate. ${ }^{\alpha} \mathrm{M} 2 \mathrm{KO} \mathrm{Ctrl}<\mathrm{M} 2 \mathrm{WT}$ Ctrl (normoxia); significant by two-sample t-test, $\mathrm{P}<0.05$. ${ }^{\beta} \mathrm{M} 2 \mathrm{KO}$ Ctrl $<\mathrm{M} 2 \mathrm{WT}$ Ctrl (hypoxia); significant by two-sample t-test, $\mathrm{P}<0.05$. ${ }^{\chi} \mathrm{M} 2 \mathrm{KO}$ LTag $>\mathrm{M} 2 \mathrm{WT}$ LTag (normoxia); significant by one-sample t-test, $\mathrm{P}<0.05 .{ }^{\circ} \mathrm{M} 2 \mathrm{KO}$ LTag $>$ M2WT LTag (hypoxia); significant by one-sample t-test, $\mathrm{P}<0.05$.
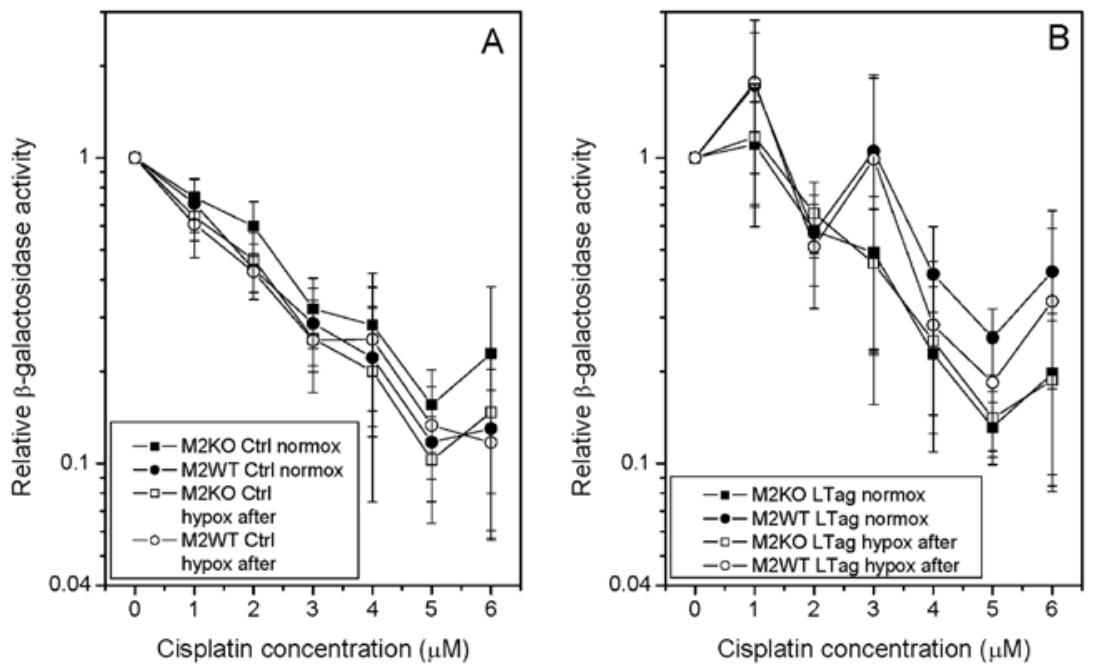

Figure 5. Effects of LTag expression on host cell reactivation of $\beta$-galactosidase activity for the cisplatin-damaged AdCA35lacZ virus in MSH2-proficient M2WT and MSH2-deficient M2KO primary murine fibroblasts under normoxic (closed symbols) or hypoxic (open symbols) incubation conditions. (A) MSH2-deficient M2KO pBabe-neo control cells and MSH2-proficient M2WT pBabe-neo control cells. (B) MSH2-deficient M2KO pBabe-neo Large Tag cells and MSH2-proficient M2WT pBabe-neo Large Tag cells. Each point represents the average \pm SE of 5 independent experiments each performed in triplicate.

compared to mMSH2-proficient murine fibroblasts transfected with the plasmid expressing SV40 large T antigen under both normoxic and hypoxic conditions (Fig. 4B).

Effects of SV4O large T antigen expression on host cell reactivation in MSH2-deficient and MSH2-proficient MEFs. We also examined HCR of the cisplatin-damaged reporter gene in primary mMSH2-deficient and mMSH2-proficient murine fibroblasts that had been transfected with a plasmid expressing the SV40 large T antigen. Consistent with the MTT assay results for non-transfected primary murine fibroblasts
(Fig. 2B), HCR was not significantly different in primary mMSH2-deficient compared to mMSH2-proficient murine fibroblasts transfected with the control plasmid (Fig. 5A). In addition, HCR was not significantly different in primary mMSH2-deficient compared to mMSH2-proficient murine fibroblasts transfected with the plasmid expressing SV40 large $\mathrm{T}$ antigen (Fig. 5B).

Effects of hypoxia. We were unable to detect any significant effect of hypoxia on cisplatin sensitivity or HCR in primary mMSH2-deficient and mMSH2-proficient murine fibroblasts 
or in primary mMSH2-deficient and mMSH2-proficient murine fibroblasts transfected with either a control plasmid or a plasmid expressing the SV40 large T antigen. In contrast, hypoxia resulted in a decrease in HCR in SV40-transformed mMSH2-deficient MS5-7 cells but not in SV40-transformed mMSH-proficient BC1-6 cells (Fig. 2A). In addition, hypoxia did not increase the cisplatin sensitivity of SV40-transformed mMSH2-deficient MS5-7 cells or SV40-transformed mMSH2proficient BC1-6 cells as measured by the clonogenic (Fig. 1A) or the MTT assay (Fig. 1B).

\section{Discussion}

Host cell reactivation and MTT reduction capacity in MSH2deficient and MSH2-proficient MEFs. We were able to demonstrate significantly greater HCR of the cisplatin-damaged reporter gene and significantly reduced cisplatin sensitivity in transformed mMSH2-deficient MS5-7 cells compared to mMSH2-proficient BC1-6 cells under both normoxic and hypoxic conditions. In contrast, primary mMSH2-deficient M2KO fibroblasts showed a significant increase in cisplatin sensitivity compared to mMSH2-proficient M2WT fibroblasts under both normoxic and hypoxic conditions. In addition, we were unable to demonstrate a difference in HCR between primary mMSH2-deficient M2KO cells and mMSH2-proficient M2WT fibroblasts. These results indicate that loss of mMSH2 alone does not lead to increased repair of cisplatin-damaged DNA and cellular resistance to cisplatin in MEFs. Other studies also report a weak or no association between MMR deficiency and cisplatin resistance in MEFs $(13,14)$. These studies suggest that specific concurrent alterations in MMR-deficient cells may be necessary to confer resistance. A particularly convincing piece of evidence for this idea comes from two studies which both used mMSH2-deficient and mMSH2-proficient murine embryonic stem cells but showed conflicting results. One study found that the mMSH2-deficient cells were significantly more resistant to cisplatin than mMSH2-proficient controls (10) whereas, the other study showed no differences in the responses of the mMSH2-deficient and mMSH2-proficient cells to cisplatin (13).

SV40-transformation can lead to cell-specific alterations that may contribute to alterations in cisplatin sensitivity. Large $\mathrm{T}$ antigen, a protein product of simian virus 40 , binds to the tumour suppressor proteins $\mathrm{p} 53$ and $\mathrm{pRb}$ preventing their interaction with other target molecules in the cell. In contrast, $\mathrm{p} 53$ and $\mathrm{pRb}$ are fully functional in primary cells. SV40-transformed cells are also able to bypass cell cycle controls and proliferate indefinitely whereas primary cells can only be passaged a limited number of times before undergoing senescence. Studies investigating the role of MMR proteins in resistance to cisplatin have pointed to abrogated p53 as a possible candidate co-alteration required for resistance. In fact, it has been suggested that defects in p53 may make a more important contribution to resistance than defects in MMR $(14,25)$. In the context of the current work, it appears likely that the combination of SV40-transformation-induced changes (i.e., possibly stemming from altered p53 activity) and $\mathrm{MSH} 2$ deficiency in MS5-7 cells results in enhanced repair of cisplatindamaged DNA and a corresponding increase in resistance to cisplatin. In contrast, primary M2KO cells, which are also
MSH2-deficient, lack the requisite concomitant alterations to increase repair and confer cisplatin resistance.

In order to further examine the possible role of SV40-transformation in the cisplatin resistance of mMSH2-deficient MEFs, we examined HCR of the cisplatin-damaged reporter gene and cisplatin sensitivity by the MTT assay in primary mMSH2deficient and mMSH2-proficient murine fibroblasts transfected with a plasmid expressing SV40 large T antigen under both normoxic and hypoxic conditions. Consistent with our results for primary MEFs, the sensitivity to cisplatin was significantly greater in primary mMSH2-deficient compared to $\mathrm{mMSH} 2$ proficient murine fibroblasts transfected with the control plasmid under both normoxic and hypoxic conditions. In contrast, cisplatin sensitivity was not significantly greater in primary $\mathrm{mMSH} 2$-deficient compared to $\mathrm{mMSH} 2$-proficient murine fibroblasts transfected with the plasmid expressing SV40 large $T$ antigen under both normoxic and hypoxic conditions. In addition, HCR was not significantly different in primary mMSH2-deficient compared to $\mathrm{mMSH} 2$-proficient fibroblasts transfected with either the plasmid expressing SV40 large T antigen or the control plasmid. These results indicate that expression of the SV40 large T antigen reduces the cisplatin sensitivity of mMSH2-deficient cells relative to that of mMSH2-proficient cells. However, the increase in the relative cisplatin resistance is not concomitant with a relative increase in HCR in mMSH2-deficient cells. This suggests that the increased cisplatin resistance in the large $\mathrm{T}$ antigenexpressing mMSH2-deficient cells may result from changes other than increased DNA repair or from a change in a DNA repair pathway not detected by the HCR assay. Several studies have pointed to the importance of the homologous recombination repair (HRR) machinery in cellular responses to cisplatin $(26,27)$. Using an adenovirus based HCR assay for cisplatindamaged DNA similar to that used here, we have reported previously a reduced HCR in cells from xeroderma pigmentosum patients deficient in nucleotide excision repair (NER) but no reduction in cells from patients with Fanconi's anaemia deficient in HRR (28). The HRR pathway is required for the removal of cisplatin interstrand DNA crosslinks, whereas the NER pathway is required for removal of intrastrand DNA crosslinks. These results suggest that the adenovirus based HCR assay employed here is unable to detect differences in the repair of interstrand DNA crosslinks by HRR. This idea is consistent with the results of Day et al showing that interstrand crosslinks induced in the adenovirus genome by psoralen plus near ultraviolet light treatment are not repaired following the infection of normal human cells (29). It is therefore possible that the increased cisplatin resistance in the SV40-transformed and transfected $\mathrm{mMSH} 2$-deficient cells expressing large $\mathrm{T}$ antigen, compared to their transformed and transfected mMSH2proficient counterparts results, in part at least, from increased HRR.

It has been suggested that the MSH2 gene product protects cells from tumourigenesis by facilitating apoptosis and p53 activation (30). Therefore, another possible change leading to increased cisplatin resistance in the large $\mathrm{T}$ antigen-expressing mMSH2-deficient cells is reduced apoptosis. In support of this argument, studies employing p53-deficient Li-Fraumeni cells have suggested that $\mathrm{p} 53$ 's role in regulating apoptosis may be a more important determinant of cell fate than its role 
in NER (31). Accordingly, while loss of p53 function may enable cells to survive cisplatin damage, a greater mutagenic phenotype will result from the accumulated DNA damage. Since enhanced HCR of the cisplatin-damaged reporter gene was not observed in mMSH2-deficient compared to mMSH2proficient fibroblasts transfected with the plasmid expressing large $\mathrm{T}$ antigen, yet enhanced HCR was observed in SV40transformed mMSH2-deficientcompared to mMSH2-proficient fibroblasts, this suggests the involvement of additional genetic alterations in the SV40-transformed cells subsequent to the initial introduction of large $\mathrm{T}$ antigen.

Survival of cisplatin-treated SV40-transformed MEFs. HCR of the cisplatin-damaged reporter gene was significantly enhanced in mMSH2-deficient MS5-7 cells relative to mMSH2-proficient BC1-6 cells. In the clonogenic survival assay we have also shown significantly enhanced survival of MS5-7 cells compared to BC1-6 cells. Similar results have been reported for these cell lines in response to ionizing radiation $(22,30)$. Taken together, these results suggest that elevated NER contributes to cisplatin resistance in MS5-7 cells. Other reports have similarly implicated enhanced DNA repair as a key mechanism of cisplatin resistance in murine and human tumour cells $(21,32)$.

Effects of hypoxia. HCR in SV40-transformed mMSH2deficient MS5-7 cells was reduced in hypoxia. This finding suggests that repair of cisplatin-damaged DNA is reduced in MSH2-deficient MS5-7 cells under conditions of hypoxia. In contrast, the cisplatin sensitivity of mMSH2-deficient MS5-7 cells was not changed under hypoxic versus normoxic conditions. Increased genetic instability, hypermutability and decreased repair of UV-induced DNA damage have been previously reported in MMR-deficient cells exposed to hypoxic stress (16). Taken together, these results suggest that the reduced repair of cisplatin-damaged DNA in hypoxia with no corresponding reduction in cell survival following cisplatin treatment in mMSH2-deficient MS5-7 cells may favour the accumulation of mutations resulting in genetic instability and a more aggressive tumour phenotype. This identifies a possible mechanism by which genetic instability may be increased in MMR-deficient cells under hypoxic conditions.

\section{Acknowledgements}

This work was supported by an operating grant from the National Cancer Institute of Canada with funds from the Canadian Cancer Society (grant no. 16066).

\section{References}

1. Siddik ZH: Cisplatin: mode of cytotoxic action and molecular basis of resistance. Oncogene 22: 7265-7279, 2003.

2. Fan S, el-Deiry WS, Bae I, Freeman J, Jondle D, Bhatia K, Fornace AJ Jr, Magrath I, Kohn KW and O'Connor PM: p53 gene mutations are associated with decreased sensitivity of human lymphoma cells to DNA damaging agents. Cancer Res 54: 5824-5830, 1994.

3. Segal-Bendirdjian E, Mannone L and Jacquemin-Sablon A: Alteration in p53 pathway and defect in apoptosis contribute independently to cisplatin resistance. Cell Death Differ 5: 390-400, 1998.
4. Lin $X$ and Howell SB: DNA mismatch repair and p53 function are major determinants of the rate of development of cisplatin resistance. Mol Cancer Ther 5: 1239-1247, 2006.

5. Fan S, Smith ML, Rivet DJ, II, Duba D, Zhan Q, Kohn KW, Fornace AJ Jr and O'Connor PM: Disruption of p53 function sensitizes breast cancer MCF-7 cells to cisplatin and pentoxifylline. Cancer Res 55: 1649-1654, 1995.

6. Hawkins DS, Demers GW and Galloway DA: Inactivation of p53 enhances sensitivity to multiple chemotherapeutic agents. Cancer Res 56: 892-898, 1996.

7. Fishel R and Kolodner R: Identification of mismatch repair genes and their role in the development of cancer. Curr Opin Genet Dev 5: 382-395, 1995.

8. Vaisman A, Varchenko M, Umar A, Kunkel T, Risinger J, Barrett C, Hamilton T and Chaney G: The role of hMLH1, hMSH3 and hMSH6 defects in cisplatin and oxaliplatin resistance: correlation with replicative bypass of platinum-DNA adducts. Cancer Res 58: 3579-3585, 1998.

9. Fink D, Nebel S, Abei S, Zheng H, Cenni B, Nehmé A, Christen R and Howell S: The role of DNA mismatch repair in platinum drug resistance. Cancer Res 56: 4881-4886, 1996.

10. Fink D, Zheng H, Nebel S, Norris P, Abei S, Lin T, Nehmé A, Christen R, Haas M, MacLeod C and Howell S: In vitro and in vivo resistance to cisplatin in cells that have lost DNA mismatch repair. Cancer Res 57: 1841-1845, 1997.

11. Fink D, Nebel S, Aebi S, Nehmé A and Howell S: Loss of DNA mismatch repair due to knockout of MSH2 or PMS2 results in resistance to cisplatin and carboplatin. Int J Oncol 11: 539-542, 1997.

12. Cenni B, Kim H, Bubley G, Abei S, Fink D, Teicher B, Howell S and Christen R: Loss of DNA mismatch repair facilitates reactivation of a reporter plasmid damaged by cisplatin. Br J Cancer 80: 699-704, 1999.

13. Claij N and Riele H: Msh2 deficiency does not contribute to cisplatin resistance in mouse embryonic stem cells. Oncogene 23: 260-266, 2004

14. Sansom O and Clarke A: The ability to engage enterocyte apoptosis does not predict long-term crypt survival in $p 53$ and Msh2 deficient mice. Oncogene 21: 5934-5939, 2002.

15. Mihaylova V, Bindra R, Yuan J, Campisi D, Narayanan L, Jensen R, Giordano F, Johnson R, Rockwell S and Glazer P: Decreased expression of the DNA mismatch repair gene $M l h l$ under hypoxic stress in mammalian cells. Mol Cell Biol 23: 3265-3273, 2003

16. Yuan J, Narayanan N, Rockwell S and Glazer P: Diminished DNA repair and elevated mutagenesis in mammalian cells exposed to hypoxia and low pH. Cancer Res 60: 4372-4376, 2000.

17. Rainbow AJ: Defective repair of UV-damaged DNA in human tumor and SV40-transformed human cells but not in adenovirustransformed human cells. Carcinogenesis 10: 1073-1077, 1989.

18. Bowman K, Sicard D, Ford J and Hanawalt P: Reduced global genomic repair of ultraviolet light-induced cyclobutane pyrimidine dimers in simian virus 40-transformed human cells. Mol Carcinog 29: 17-24, 2000.

19. Digweed M, Demuth I, Rothe S, Scholz R, Jordan A, Grötzinger C, Schindler D, Grompe M and Sperling K: SV40 large T-antigen disturbs the formation of nuclear DNA-repair foci containing MRE11. Oncogene 21: 4873-4878, 2002.

20. Hein J, Boichuk S, Wu J, Cheng Y, Freire R, Jat PS, Roberts TM and Gjoerup OV: Simian virus 40 large T antigen disrupts genome integrity and activates a DNA damage response via Bubl binding. J Virol 83: 117-127, 2009.

21. Chu G: Cellular responses to cisplatin. J Biol Chem 269: 787-790, 1994.

22. Fritzell J, Narayanan L, Baker S, Bronner C, Andrew S, Prolla T, Bradley M, Jirik F, Liskay R and Glazer P: Role of DNA mismatch repair in the cytotoxicity of ionizing radiation. Cancer Res 57: 5143-5147, 1997.

23. Addison C, Hitt M, Kunsken D and Graham F: Comparison of the human versus murine cytomegalovirus immediate early gene promoters for transgene expression by adenoviral vectors. J Gen Virol 78: 1653-1661, 1997.

24. Sambrook J, Fritsch EF and Maniatis T: Standard protocol for calcium phosphate-mediated transfection of adherent cells. In: Molecular Cloning: A Laboratory Manual. 2nd edition. Cold Spring Harbor Laboratory Press, New York, pp16.33-16.36, 1989.

25. Branch P, Masson M, Aquilina G, Bignami M and Karran P: Spontaneous development of drug resistance: mismatch repair and p53 defects in resistance to cisplatin. Oncogene 19:3138-3145, 2000 . 
26. Panni E, Stojic L, El-Shemerly M, Jiricny J and Ferrari S: Mismatch repair status and the response of human cells to cisplatin. Cell Cycle 6: 1796-1802, 2007.

27. Zhen W, Link C Jr, O'Connor P, Reed E, Parker R, Howell S and Bohr V: Increased gene-specific repair of cisplatin interstrand crosslinks in cisplatin-resistant human ovarian cancer cell lines. Mol Cell Biol 12: 3689-3698, 1992.

28. Davis K, Tomkins D and Rainbow AJ: Roberts syndrome fibroblasts showing cisplatin hypersensitivity have normal host cell reactivation of cisplatin-treated adenovirus and normal capacity of cisplatin-treated cells for adenovirus DNA synthesis. Soma Cell Mol Genet 22: 393-402, 1996.

29. Day RS, Giuffrida AS and Dingman CW: Repair by human cells of adenovirus- 2 damaged by psoralen plus near ultraviolet light treatment. Mutat Res 132: 311-320, 1975.
30. Peters A, Young L, Maeda T, Tron V and Andrew SE: Mammalian DNA mismatch repair protects cells from UVB-induced DNA damage by facilitating apoptosis and p53 activation. DNA Repair 2: 427-435, 2003.

31. Bhana S and Lloyd DR: The role of p53 in DNA damage-mediated cytotoxicity overrides its ability to regulate nucleotide excision repair in human fibroblasts. Mutagenesis 23: 43-50, 2008.

32. Eastman A and Schulte N: Enhanced DNA repair as a mechanism of resistance to cis-diamminedichloroplatinum(II). Biochemistry 27: 4730-4737, 1988. 